\title{
Conjugation to PEG as a strategy to limit the uptake of drugs by the placenta: potential applications for drug administration in pregnancy
}

Article

Published Version

Creative Commons: Attribution 4.0 (CC-BY)

Open Access

Dodd, A., Natfji, A. A. ORCID: https://orcid.org/0000-00026168-4632, Evangelinos, A., Grigoletto, A., Pasut, G., Beards, F., Renshall, L., Osborn, H. M. I., Greco, F. and Harris, L. K. (2022) Conjugation to PEG as a strategy to limit the uptake of drugs by the placenta: potential applications for drug administration in pregnancy. Molecular Pharmaceutics, 19 (1). pp. 345-353. ISSN 1543-8392 doi:

https://doi.org/10.1021/acs.molpharmaceut.1c00498 Available at https://centaur.reading.ac.uk/101866/

It is advisable to refer to the publisher's version if you intend to cite from the work. See Guidance on citing.

To link to this article DOI: http://dx.doi.org/10.1021/acs.molpharmaceut.1c00498

Publisher: American Chemical Society

All outputs in CentAUR are protected by Intellectual Property Rights law, including copyright law. Copyright and IPR is retained by the creators or other copyright holders. Terms and conditions for use of this material are defined in 
the End User Agreement.

www.reading.ac.uk/centaur

\section{CentAUR}

Central Archive at the University of Reading

Reading's research outputs online 


\title{
Conjugation to PEG as a Strategy to Limit the Uptake of Drugs by the Placenta: Potential Applications for Drug Administration in Pregnancy
}

\author{
Abbie Dodd, ${ }^{\ddagger}$ Az Alddien Natfji, ${ }^{\ddagger}$ Angelos Evangelinos, ${ }^{\ddagger}$ Antonella Grigoletto, Gianfranco Pasut, \\ Frances Beards, Lewis Renshall, Helen M. I. Osborn, Francesca Greco, * and Lynda K. Harris*
}

Cite This: https://doi.org/10.1021/acs.molpharmaceut.1c00498

Read Online

ABSTRACT: Here, we evaluated the feasibility of non-prodrug PEG-drug conjugates to decrease the accumulation of drugs within the placental tissues. The results showed that PEG was biocompatible with the human placenta with no alteration of the basal rate of proliferation or apoptosis in term placental explants. No significant changes in the released levels of lactate dehydrogenase and the human chorionic gonadotropin were observed after PEG treatment. The cellular uptake studies revealed that conjugating Cy5.5 and haloperidol to PEG significantly reduced (by up to $\sim 40$-fold) their uptake by the placenta. These findings highlight the viability of novel non-prodrug polymer-drug conjugates to avoid the accumulation of drugs within the placenta.

KEYWORDS: polymer-drug conjugate, PEG, pregnancy, placenta, transport, drug delivery

\section{INTRODUCTION}

The usage of teratogenic medicines in pregnancy presents many challenges due to the intrinsic risk that they may cross the placenta barrier and be harmful to the developing fetus. Furthermore, the development of new drugs suffers from a paradox. ${ }^{1}$ On the one hand, each drug needs to be risk assessed on a case-by-case basis. On the other hand, obtaining clinical safety data for the administration of even routine drugs during pregnancy is very difficult, as the default position is to err on the side of caution and avoid most medicines altogether, unless absolutely necessary. ${ }^{2}$ Therefore, a technology that restricts the therapeutic effects of drugs to the maternal bloodstream would be beneficial both for the expectant mother and to protect the fetus from toxic effects. ${ }^{3}$

The conjugation of drugs to polymers has been previously employed in an attempt to develop a drug delivery system able to restrict the distribution of a fluorescent dye to the maternal compartment. ${ }^{4}$ In that study, poly(amidoamine) (PAMAM) (G4-OH) dendrimers were used as nanocarriers to which Alexa (488) dye was conjugated. The results indicated a low transfer of the PAMAM-Alexa (488) conjugate compared to the free dye ${ }^{4}$ using an ex vivo model. However, concerns regarding the safety profiles of dendritic PAMAMs (based on their generation, charge, and concentration) may limit the therapeutic applications of these systems. ${ }^{5}$ Although PAMAM G4-OH has been shown to be well tolerated in vivo, ${ }^{6}$ their unpredictable pharmacokinetics and variable characterization profiles (fluctuating purity and reproducibility) make them less attractive for their design and development for treating complications during pregnancy. 7,8

We recently disclosed a PEGylated polymer-drug conjugate that can prevent the diffusion of a drug through the blood-brain barrier while retaining its therapeutic effects outside of the central nervous system. ${ }^{9,10}$ More specifically, we demonstrated that conjugation of a drug (the dopamine receptor antagonist haloperidol) to PEG, through a nonbiodegradable linker, allowed the drug to retain affinity for the receptor (albeit reduced) and prevented it from crossing the blood-brain barrier.

In this Communication, we propose and validate the concept of PEG-drug conjugation via a nondegradable linker as a means to restrict drugs to the maternal bloodstream, thus preventing fetal transfer (Figure 1). This hypothesis is assessed at three levels. First, we evaluate the biocompatibility of PEG with the placental tissue by measuring changes in the proliferation, apoptosis, necrosis, and the secretory function in human term placental explants after exposure to PEG. Then, using a fluorescently tagged PEG conjugate (PEG-cyanine-5.5 (Cy5.5)), we probe the ability of conjugation to prevent cellular uptake of the free Cy5.5 dye by the placenta. Finally, we validate the hypothesis by measuring the placental uptake of PEG-drug conjugates that contain two different molecular weights of PEG, and a drug (haloperidol) that can freely penetrate the placental barrier and reach the fetus when administered in the nonconjugated form. ${ }^{10}$ By using an actual drug, we, therefore, confer a clinical meaning to the proposed strategy.

Received: June 22, 2021

Revised: November 10, 2021

Accepted: November 11, 2021 


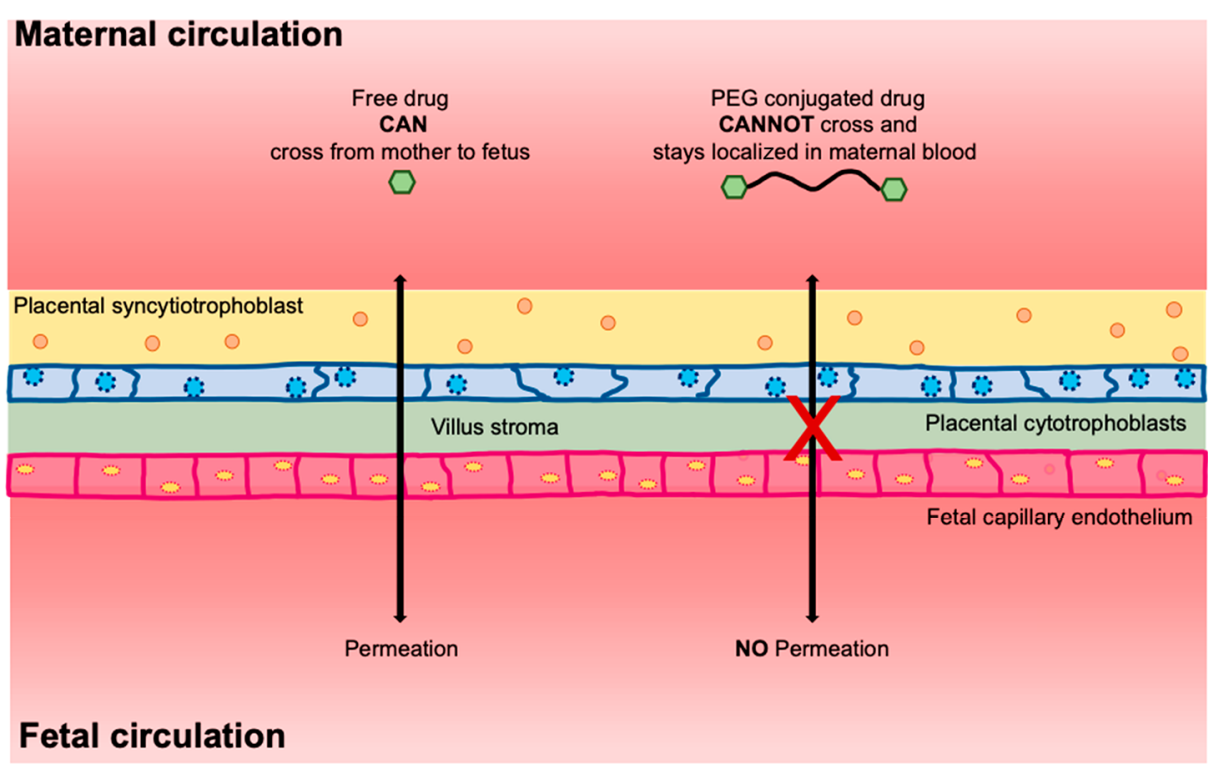

Figure 1. Cartoon representation of:(i) the distribution of a free small molecule drug into the fetal compartment after systemic administration into maternal circulation and (ii) restricted distribution of the PEG-conjugated drug within the maternal compartment after its systemic administration in maternal circulation.

\section{MATERIALS AND METHODS}

Materials. $\alpha$ - and $\omega$-Disuccinimidyl ester poly(ethylene glycol) (molecular weights of 6000 and $2000 \mathrm{Da}$ ) and $\alpha$ methoxy-omega-amino poly(ethylene glycol) (molecular weight $5000 \mathrm{Da}$ ) were obtained from Iris Biotech $\mathrm{GmbH}$, Germany. Cy5.5-NHS ester was purchased from GE Healthcare (UK). Haloperidol was obtained from Sigma-Aldrich, UK. All other solvents and chemicals were purchased from Fisher Scientific and Sigma-Aldrich, UK, and were used without any further purification steps unless otherwise stated.

Methods. Synthesis and Characterization of PEG-Cy5.5 and PEG-Haloperidol Conjugates. The synthesis and characterization of the novel PEG-Cy5.5 conjugate was carried out as follows. $\mathrm{PEG}-\mathrm{NH}_{2}(4.37 \mathrm{mg}, 0.874 \mu \mathrm{mol})$ was solubilized in $300 \mu \mathrm{L}$ of $0.1 \mathrm{M} \mathrm{Na}_{2} \mathrm{HPO}_{4}, 0.15 \mathrm{M} \mathrm{NaCl}, \mathrm{pH}$ 8.0, and 1 equiv of dye, previously dissolved in anhydrous DMSO at a concentration of $10 \mathrm{mg} / \mathrm{mL}$. The reaction was stirred overnight at room temperature in the dark and was purified by size exclusion chromatography (SEC)-HPLC with an analytical Zorbax GF-250 column $(250 \times 4.6 \mathrm{~mm})$ that was eluted with $20 \mathrm{mM} \mathrm{Na} \mathrm{HPO}_{4}$ and $130 \mathrm{mM} \mathrm{NaCl}(\mathrm{pH} 7.2)$ containing $20 \%(\mathrm{v} / \mathrm{v})$ of ACN at a flow rate of $0.3 \mathrm{~mL} / \mathrm{min}$. The effluent was monitored by measuring the absorbance at $675 \mathrm{~nm}$. PEG-Cy5.5 was purified from the unreacted dye through Pierce Dye Removal Columns, and the purity of the products was confirmed by SEC-HPLC. After the solution was dialyzed against water to remove DMSO, the product was lyophilized. The amount of Cy5.5 was determined spectrophotometrically at the maximum absorption of $694 \mathrm{~nm}$ (molar extinction coefficient: $250000 \mathrm{M}^{-1} \mathrm{~cm}^{-1}$ ). The synthesis and characterization of PEG-haloperidol conjugates (2000 and $6000 \mathrm{Da}$ ) was performed according to our previously reported studies. ${ }^{9,10}$ Specifically, herein, we have extended the previously reported synthesis of PEG-haloperidol conjugate $(6000 \mathrm{Da})$ to a different molecular weight PEG (2000 Da).

Human Tissue Collection. Healthy term placentas (37-42 week gestation) were collected from normal pregnancies within 30 min of elective Caesarean sections in accordance with North
West Local Research Ethical Committee approval (REC 15/ NW/0829); written informed consent was obtained from all women.

Placental Explant Tissue Culture. Samples of villous placental tissue were randomly collected, washed with serum free medium, and dissected into $3 \mathrm{~mm}^{3}$ explants under sterile conditions. Explants were harvested and established in culture at $0 \mathrm{~h}$ and were treated immediately for 24 to $48 \mathrm{~h}$ at $37^{\circ} \mathrm{C}$ in $5 \%$ $\mathrm{CO}_{2}{ }^{11}$ as a way to preserve the placental phenotype. In our hands, we have not observed any significant shedding of the syncytiotrophoblast during this time frame; thus, we consider it a robust model for short-term treatment studies. ${ }^{11-14}$ Placental explants were cultured in a 1:1 ratio of DMEM and Ham's F12 media (Lonza Biosciences, UK) supplemented with $10 \%(\mathrm{v} / \mathrm{v})$ fetal bovine serum (Life Technologies), L-glutamine ( $2 \mathrm{mmol} /$ $\mathrm{L})$, streptomycin $(100 \mu \mathrm{g} / \mathrm{mL})$, penicillin $(100 \mathrm{IU} / \mathrm{mL})$, and amphotericin B $(2.5 \mathrm{mg} / \mathrm{mL})$ (Invitrogen) in 24 well culture plates precoated with agarose $(1 \%(\mathrm{w} / \mathrm{v})$; Sigma-Aldrich).

Cytotoxicity Assessment with Free PEG. Placental explants were incubated with PEG-COOH (6000 Da, called free PEG throughout the paper) dissolved in $0.5 \% \mathrm{DMSO}$ in sterile PBS at a concentration range of $0-2 \mathrm{mg} / \mathrm{mL}$ for 24 or $48 \mathrm{~h}$. Samples of culture media were collected in triplicate and stored at $-20^{\circ} \mathrm{C}$ to quantify lactate dehydrogenase (LDH) release and hCG secretion. Explants were treated with $0.3 \mathrm{M} \mathrm{NaOH}$ for protein quantification or fixed in $4 \%(\mathrm{v} / \mathrm{v})$ neutral buffered formalin ( $\mathrm{pH}$ 7.4) overnight, washed in PBS, dehydrated, and paraffinembedded. The final concentration of DMSO that the explants were exposed to was $\leq 0.25 \%$. We have previously shown that concentrations of DMSO of $0.6 \%(\mathrm{v} / \mathrm{v})$ do not alter basal rates of proliferation, apoptosis, and nutrient transport in human term placental explants. ${ }^{15}$ Therefore, we did not include a DMSO vehicle control in these experiments.

Protein Assay. Placental explants were digested with $0.3 \mathrm{M}$ $\mathrm{NaOH}$ or homogenization in an ice-cold $1.15 \% \mathrm{KCl}$ solution. Total protein (mg) per explant was quantified using a Bio-Rad protein assay (Bio-Rad Laboratories, UK) according to the manufacturer's instructions and compared to a standard curve prepared with bovine serum albumin. 
Immunohistochemistry. Placental tissue sections $(\sim 5 \mu \mathrm{m})$ were deparaffinized in Histoclear and ethanol and then rehydrated in $\mathrm{ddH}_{2} \mathrm{O}$. For antigen retrieval, slides were microwaved in sodium citrate $(0.01 \mathrm{~mol} / \mathrm{L})$, supplemented with $0.05 \%(\mathrm{v} / \mathrm{v})$ Tween 20 ( $\mathrm{pH}$ 6.0) for $10 \mathrm{~min}$. After cooling, tissue sections were incubated with $3 \%(\mathrm{w} / \mathrm{v})$ hydrogen peroxidase for $10 \mathrm{~min}$ to block the activity of endogenous peroxides, followed by washes $(2 \times$ for $5 \mathrm{~min}$ ) in Tris-buffered saline (TBS). To block nonspecific antibody binding, tissue sections were incubated with nonimmune block (2\% human serum (Dako) and 10\% goat serum (Dako) in TBS-Tween $(0.1 \% \mathrm{v} / \mathrm{v}))$ for $30 \mathrm{~min}$ at room temperature. Slides were then incubated with the following primary antibodies: mouse antihuman M30 CytoDEATH (1:100; Roche Diagnostics), mouse antihuman Ki67 (1:500; MIB-1 clone, Dako), or control mouse IgG (matched concentration; Sigma-Aldrich) overnight at $4{ }^{\circ} \mathrm{C}$ in a humidified chamber. Tissue sections were washed with TBS for $5 \mathrm{~min}$, followed by washes $(2 \times$ for $5 \mathrm{~min})$ with TBS-Tween $(0.6 \%)$ and finally with TBS for $5 \mathrm{~min}$. Sections were incubated with a biotinylated goat antimouse secondary antibody (1:200; Dako) for $30 \mathrm{~min}$ at room temperature and, then, washed as described above and incubated with avidin peroxidase ( $5 \mu \mathrm{g} / \mathrm{mL}$ in TBS; Sigma-Aldrich, UK) for $30 \mathrm{~min}$ at room temperature. Slides were washed again as described above and incubated for 2-3 min with the chromogen diaminobenzidine (DAB; $0.05 \%(w / v)$; Sigma-Aldrich, UK). Slides were rinsed with $\mathrm{ddHO}_{2}$, counterstained with filtered Harris' hematoxylin, and dehydrated in increasing concentrations of alcohol. Tissue sections were then passed in Histoclear and mounted in DPX mountant (Sigma-Aldrich, UK).

Immunostained explants were imaged using an Olympus BX41 microscope to assess the numbers of M30-positive and Ki67-positive cells as a measure of apoptosis and proliferation, respectively. Six random images of each explant were captured using the same exposure settings and analyzed using HistoQuest Analysis Software.

LDH Cytotoxicity Assay. LDH released into the explant culture medium was measured using a Cytotoxicity Detection $\mathrm{Kit}^{\text {Plus }}$ (Roche, UK) as per the manufacturer's instructions. Briefly, samples of media were thawed on ice, and $100 \mu \mathrm{L} /$ well was combined with $100 \mu \mathrm{L}$ of reaction solution and incubated for $15 \mathrm{~min}$ at room temperature. To quench the reaction, $50 \mu \mathrm{L}$ of stop solution was added; absorbance at $490 \mathrm{~nm}$ was measured using a plate reader (Versamax, Molecular Devices, Wokingham), and data were normalized to the total protein content $(\mathrm{mg})$ of each explant.

hCG Secretion Assay. hCG in culture media was measured using a commercially available hCG ELISA kit (DRG Instru- ments $\mathrm{GmbH}, \mathrm{Germany})$. Twenty-five $\mu \mathrm{L}$ of the control, standard, or unknown samples was added to individual wells of a microtiter plate, which had been precoated with a monoclonal antibody to the $\alpha$-chain of hCG conjugated with horseradish peroxidase. $100 \mu \mathrm{L}$ of the enzyme conjugate was added, and plates were incubated for $30 \mathrm{~min}$ at room temperature. After washing, $100 \mu \mathrm{L}$ of substrate solution was added and the plates were incubated for $10 \mathrm{~min}$ at room temperature. After the addition of $50 \mu \mathrm{L}$ of stop solution, the absorbance at $450 / 620$ $\mathrm{nm}$ was measured. hCG concentrations were determined using the obtained standard curve and normalized to total protein content of each explant.

PEG-Cy5.5 Conjugate Uptake Study. Placental explants were treated with the PEG-Cy5.5 conjugate at a range of concentrations from $10 \mu \mathrm{g} / \mathrm{mL}$ to $1 \mathrm{mg} / \mathrm{mL}$. The placental homing peptide iRGD-rhodamine (CRGDKGPDC-Rho; 20 $\mu \mathrm{M})$, which rapidly accumulates in the outer trophoblast layer of the explants, was employed as a positive control. ${ }^{11}$ The explants were incubated in the dark for 6 or $24 \mathrm{~h}$ at $37^{\circ} \mathrm{C}, 5 \% \mathrm{CO}_{2}$ and then embedded in OCT and frozen. Tissue sections $(5 \mu \mathrm{m})$ were fixed in ice cold methanol for $10 \mathrm{~min}$, washed with PBS $(2 \times$ for $5 \mathrm{~min}$ ), and mounted using Vectashield mounting medium containing DAPI (Vectorlabs, UK). Tissue uptake was assessed using a Zeiss Axio Observer fluorescence microscope (excitation, $650 \mathrm{~nm}$; emission, $670 \mathrm{~nm}$ ).

PEG-Haloperidol Conjugate Uptake Study. In 24 well plates, placental explants were incubated with either free haloperidol or the PEG-haloperidol conjugates at a final concentration of $20 \mu \mathrm{M}$ (haloperidol equivalent) for $24 \mathrm{~h}$ at 37 ${ }^{\circ} \mathrm{C}$ and $5 \% \mathrm{CO}_{2}$. The extraction of haloperidol or PEGhaloperidol from the tissue was performed according to a previously reported protocol for extracting haloperidol from biological tissues. ${ }^{16}$ Briefly, placental explants were washed 3 times with PBS and homogenized in an ice-cold 1.15\% KCI solution (3 volumes) using a Stuart-SHM1 homogenizer (10$15 \mathrm{~s}$ cycles). Samples for protein quantification were obtained, and the homogenates were then mixed and vortexed with $2 \%$ (v/ $\mathrm{v}$ ) acetic acid in methanol ( 2 volumes). The final homogenate mixture was centrifuged at $10 \mathrm{~kg}$ for $5 \mathrm{~min}$. The supernatants were collected and subjected to RP-HPLC analysis to quantify the amount of free or conjugated haloperidol in the tissue.

To validate the extraction method, explant culture medium containing haloperidol $(20 \mu \mathrm{M})$ was incubated without explants for $24 \mathrm{~h}$ under the same incubation conditions of the treated explants and was subjected to the same extraction procedure. Explant culture medium containing freshly added haloperidol $(20 \mu \mathrm{M})$ was also subjected to the same extraction procedure. The recovery (\%) was calculated using the following equation:

$$
100 \times \frac{(\text { concentration of haloperidol extracted from treatment solution after incubation })}{(\text { concentration of haloperidol extracted from treatment solution before incubation })}
$$

The mean recovery of haloperidol was $66 \%$.

RP-HPLC Analysis of Extracted Free Haloperidol and PEGHaloperidol. The RP-HPLC analysis of haloperidol was performed according to our previously reported protocol. ${ }^{10}$ The RP-HPLC analysis was carried out using a C18 column. A gradient system was applied using an aqueous $(0.25 \%$ acetic acid) (A) gradient in acetonitrile (B) (10\% of B increased to $70 \%$ of $\mathrm{B}$ over $25 \mathrm{~min}$ and then decreased to $10 \%$ of $\mathrm{B}$ for 10 $\mathrm{min})$. The flow rate was adjusted to $1 \mathrm{~mL} / \mathrm{min}$, and the injection volume was $20 \mu \mathrm{L}$. The system was run for $40 \mathrm{~min}$, and the eluant was detected using a UV detector at $\lambda=245 \mathrm{~nm}$. A stock solution of haloperidol $(1 \mathrm{mg} / \mathrm{mL})$ in $2 \%$ acetic acid in methanol was prepared and used to obtain a range of concentrations $(0.025-30 \mu \mathrm{g} / \mathrm{mL})$. A RP-HPLC calibration curve was obtained to determine the concentration of free haloperidol or PEG-haloperidol conjugates taken up by the placental explants.

Statistical Analysis. Graphical representations and analysis of the data were generated using GraphPad Prism software (Version 7). Data were either expressed as medians (for nonparametric data) or presented as mean \pm SEM (standard 
A)

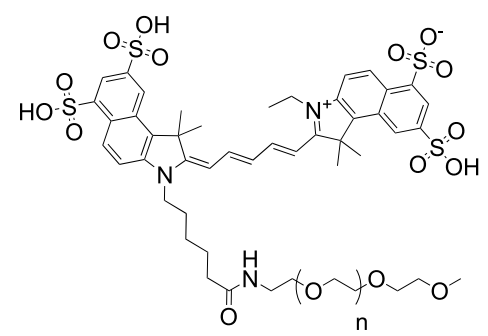

B)

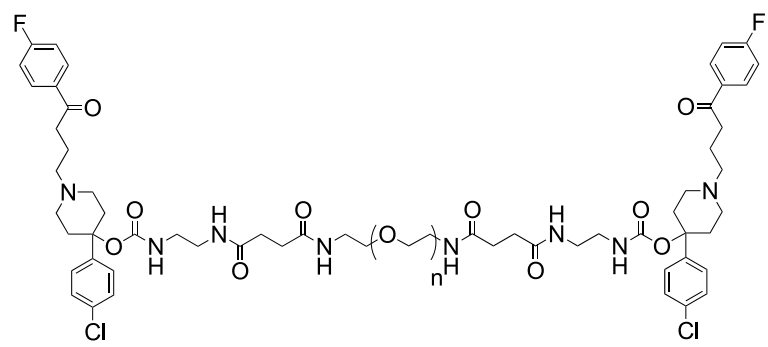

Figure 2. Chemical structures of (A) PEG-Cy5.5 and (B) PEG-haloperidol conjugates used in this study. ${ }^{10}$

A)
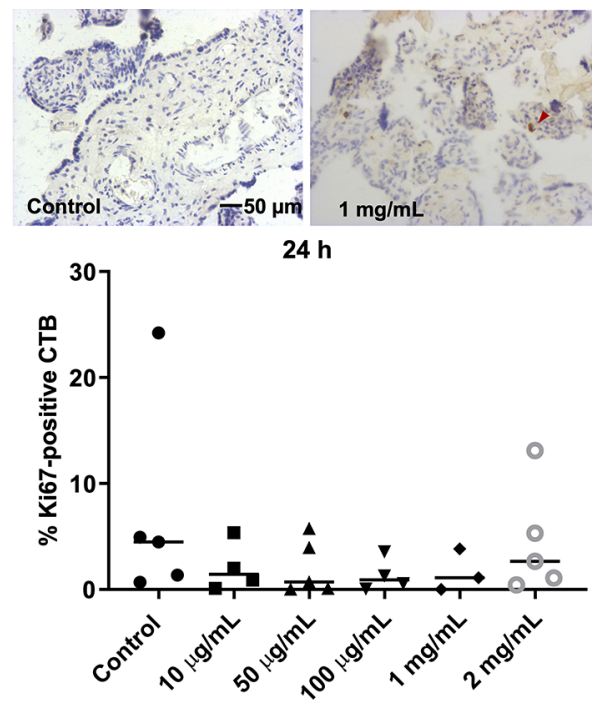

c)

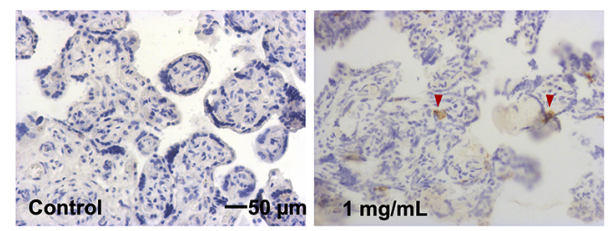

$24 \mathrm{~h}$

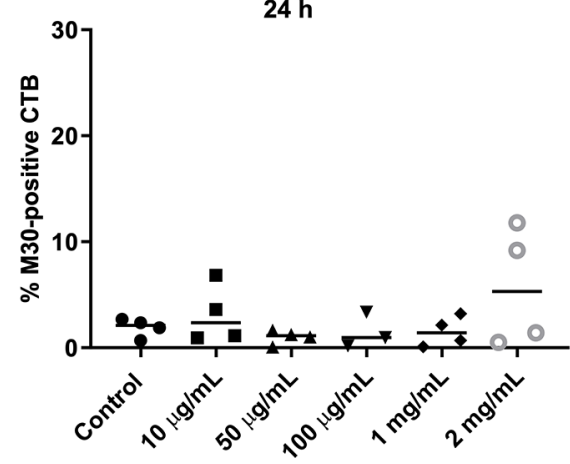

B)

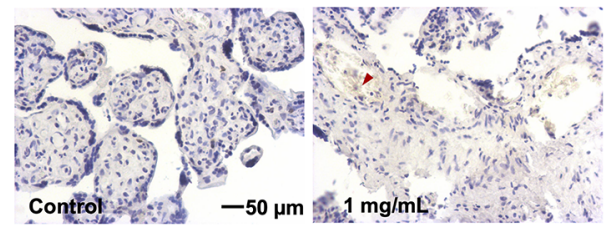

$48 \mathrm{~h}$

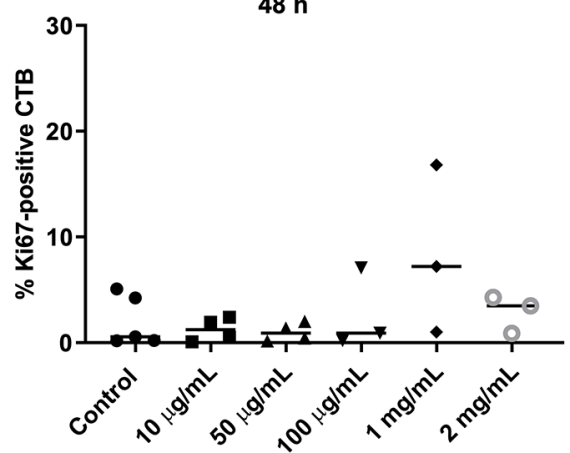

D)

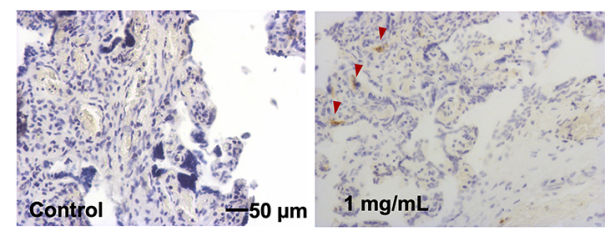

$48 \mathrm{~h}$

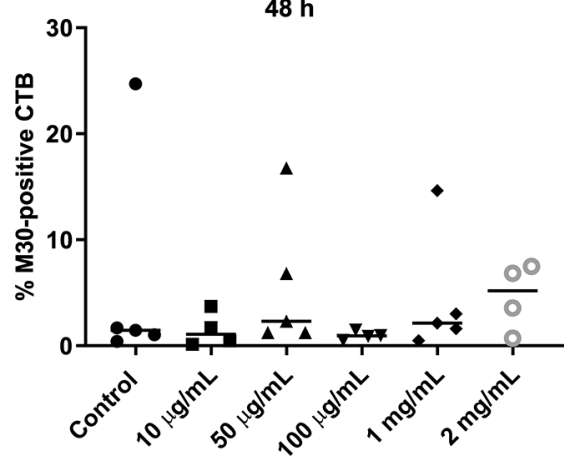

Figure 3. Evaluation of proliferation and apoptosis rates following incubation of placental explants with free PEG by immunostaining with an antibody to Ki67 and M30. (A) Quantification of Ki67-positive cells at 24 h. (B) Quantification of Ki67-positive cells at 48 h. (C) Quantification of M30-positive cells at $24 \mathrm{~h}$. (D) Quantification of M30-positive cells at $48 \mathrm{~h}$. Data presented as medians, $n \geq 3(P>0.05$, Friedman test). Images represent the evaluation of proliferation (A, B) and apoptosis (C, D) following incubation of placental explants with free PEG by immunostaining with an antibody to Ki67 or M30 (DAB; brown); nuclei were stained with hematoxylin (blue). Red arrows indicate positive staining with either Ki67 or M30. All images were captured at $20 \times$ magnification.

error of mean, $n=3-5$ ) unless stated otherwise. Statistical analysis was performed using Wilcoxon matched pairs and the Friedmann test for nonparametric data or the one-way analysis of variance (ANOVA) test followed by the Bonferroni's post hoc test where appropriate; a $P$ value of $<0.05$ was set to be significant in the parameters. The Shapiro-Wilk test was performed to detect the normal distribution of the parametric data; a $P$ value of $<0.05$ was set to be significant.

\section{RESULTS AND DISCUSSION}

PEG Conjugates Used in the Study. Three conjugates were used in this study, which contained either the Cy5.5 
A)

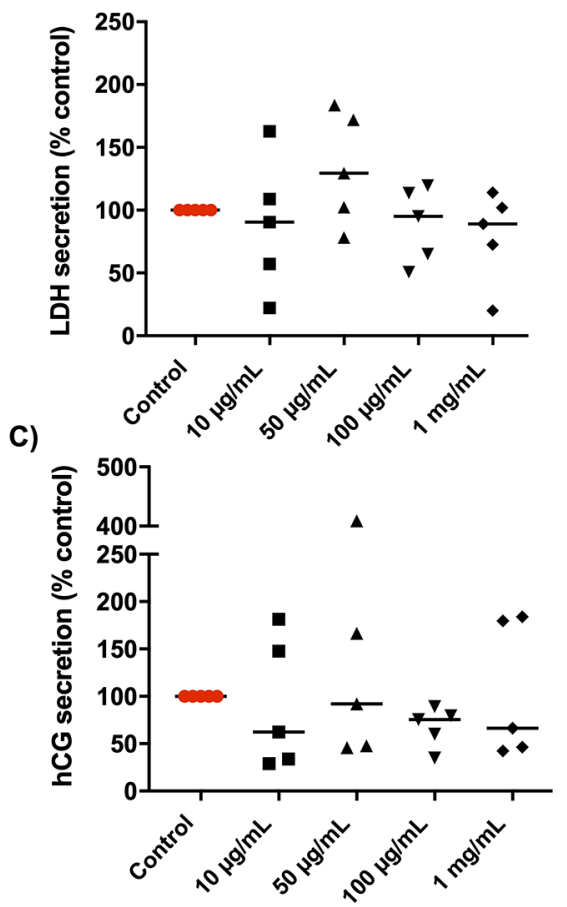

B)

D)
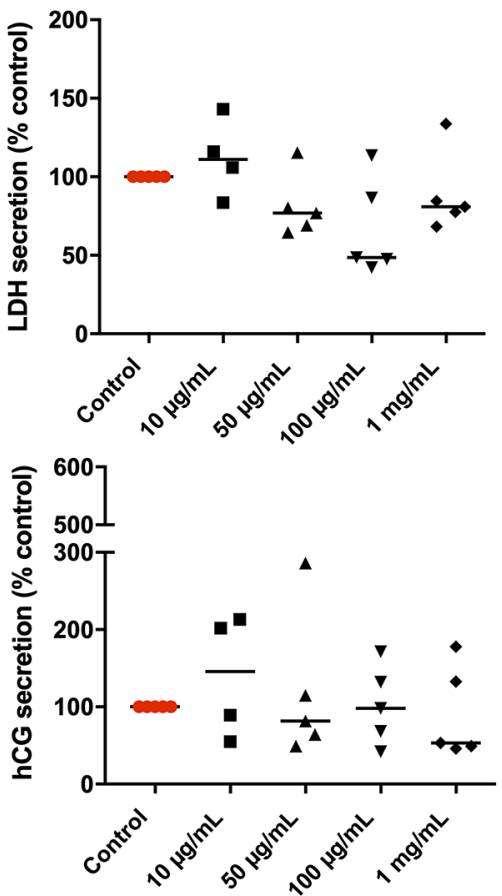

Figure 4. Quantification of LDH and hCG secretion following exposure of explants to free PEG. (A) Percentage change in LDH secretion after incubation of explants with free PEG for $24 \mathrm{~h}$. (B) Percentage change in LDH secretion after incubation of explants with free PEG for $48 \mathrm{~h}$. hCG was quantified relative to total protein content $(\mathrm{mg}$ ) of each explant. (C) Quantification of hCG release from individual explants at $24 \mathrm{~h}$. (D) Quantification of hCG release from individual explants at $48 \mathrm{~h}$. Data presented as medians, $n=5(P>0.05$, Friedman test).

fluorescent dye or the drug haloperidol. Namely, they were a PEG-Cy5.5 (5000 Da) conjugate and two PEG-haloperidol conjugates $(2000$ and $6000 \mathrm{Da}) .{ }^{10}$ These two PEG-haloperidol conjugates were chosen to assess different levels of haloperidol loading (2000 Da derivative, 25.5\% w/w; $6000 \mathrm{Da}$ derivative, $8.5 \% \mathrm{w} / \mathrm{w}$ ) to counterbalance the loss of potency observed after conjugation. ${ }^{10}$ The Cy5.5 fluorescent probe was selected to label the PEG chain due to its good solubility in biological media, its relatively high fluorescence quantum yield, and the fact that its excitation/emission spectra greatly minimize their overlap with the autofluorescence of biomolecules. Therefore, it is widely used in biological applications including placenta-related research. ${ }^{17-20}$ In addition, its chemical structure allows conjugation to PEG via an amide linkage, which is biologically stable for the intended study. ${ }^{21}$ All conjugates (both that containing the Cy5.5 dye and those containing haloperidol) were designed as non-prodrug macromolecular systems to avoid the release of the conjugated compound, which would compromise the research hypothesis, whereby released small molecules could freely accumulate within the placental tissues.

The labeling of $\mathrm{PEG}-\mathrm{NH}_{2}$ with $\mathrm{Cy} 5.5$ produced a conjugate with a dye loading of $18.47 \%(\mathrm{w} / \mathrm{w})$ and a molar ratio of Cy5.5 to PEG in the conjugate of 0.99 , as determined spectrophotometrically. The synthesis of PEG-haloperidol conjugates was carried out according to previously reported protocols, ${ }^{9,10}$ and loading of haloperidol in the conjugates was $25.5 \%$ and $8.5 \%(\mathrm{w} /$ w) for the 2000 and $6000 \mathrm{Da}$ conjugates, respectively. All conjugates had excellent purity (the free dye/haloperidol was always $<1 \% \mathrm{w} / \mathrm{w}$ of the total dye/haloperidol content). The molar ratios of haloperidol to PEG in the conjugates were also calculated to be 1.6 and 1.8 for the 6000 and $2000 \mathrm{Da}$ conjugates, respectively (Figure 2 ). ${ }^{10}$
PEG as a Polymeric Carrier Showed Biocompatibility with Human Placental Explants. PEG is an FDA approved polymer, is known to be biocompatible with various tissues, and is used for several pharmaceutical applications. ${ }^{22}$ However, as we are proposing the use of PEG-based conjugates in pregnant women, it is necessary to determine the biocompatibility of PEG with the placental tissue to ensure that it is not cytotoxic and does not alter the secretory function of the placenta. This was essential as any potential toxic effects induced by the polymeric carrier would alter the normal placental function and might affect the drug uptake and transplacental transfer. ${ }^{23}$ Therefore, it was important at the beginning of the study to evaluate the potential toxic effects of the carrier (PEG $6000 \mathrm{Da}$ ) on the placental explants. It is important to highlight that several PEGylated nanoparticles have been developed and studied for their toxicity as transplacental drug delivery systems. ${ }^{24}$ However, to the best of our knowledge, this is the first report on the biocompatibility of PEG (as a free polymer) specifically with the placental tissues.

To assess the cellular turnover post-treatment with free PEG, explants of villous placental tissue were incubated with a range of concentrations of free PEG for 24 or $48 \mathrm{~h}$. After incubation, the proliferation and apoptosis rates within the tissues were determined by immunostaining with antibodies to Ki67 and M30, respectively. Treatment with free PEG did not significantly alter the basal rates of the proliferation or apoptosis at any concentration tested (treated vs control $P>0.05$ ), nor did the duration of incubation (median for Ki67 and M30-positive cells $24 \mathrm{~h}$ vs $48 \mathrm{~h}, P>0.05)$ when compared with the untreated controls $(P>0.05)$ (Figure 3$)$. These findings are in agreement with other studies reporting no changes in the Ki67 and M30 immunoreactivity in normal placental tissues when compared to 
A)

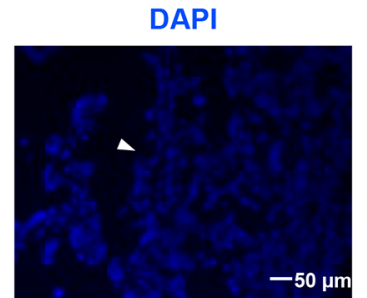

$24 \mathrm{~h}$

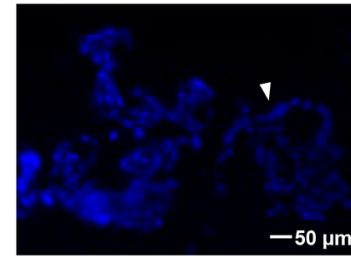

B)

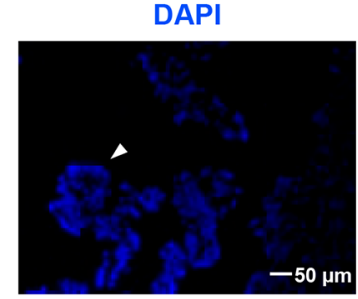

$24 \mathrm{~h}$

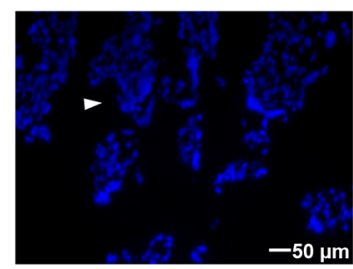

C)

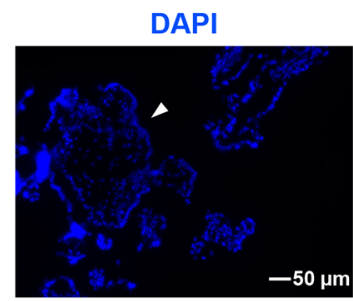

$24 \mathrm{~h}$

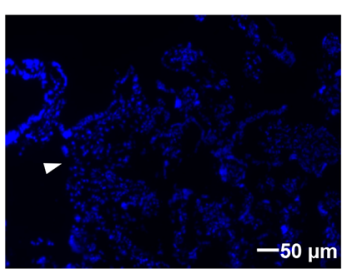

iRGD-Rho
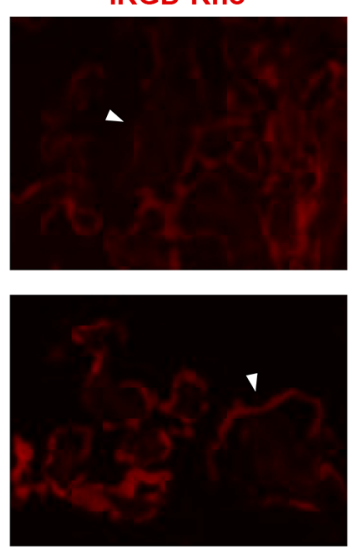

Free Cy5.5 dye
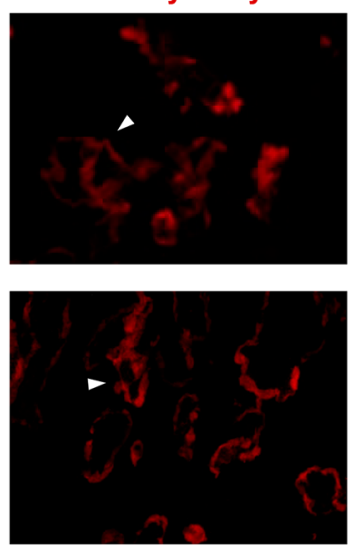

PEG-Cy5.5 conjugate
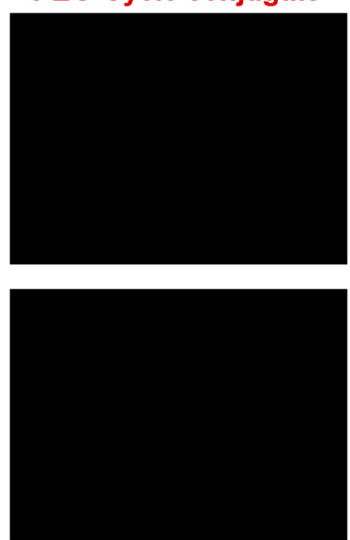
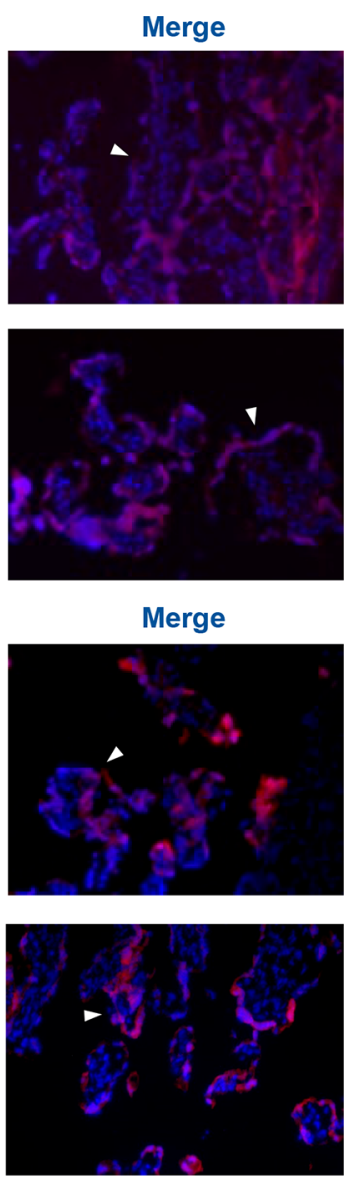

Merge
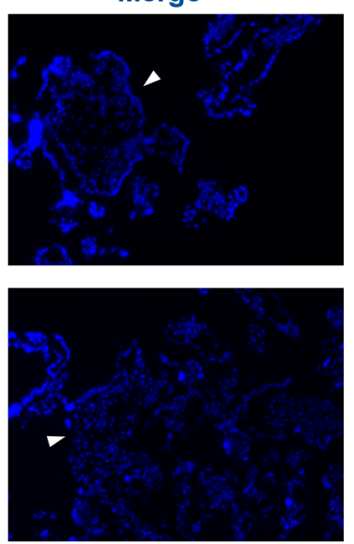

Figure 5. (A) iRGD-Rho tumor-homing peptide (red) accumulation in the outer syncytiotrophoblast layer of term placental explants after incubation for 6 or $24 \mathrm{~h}($ at $20 \mu \mathrm{M})$. (B) Free Cy5.5 dye (red) accumulation in term placental explants after incubation for 6 or $24 \mathrm{~h}($ at $2 \mu \mathrm{g} / \mathrm{mL})$. (C) PEGCy5.5 conjugate showed no placental accumulation after treatment for 6 or $24 \mathrm{~h}$. Data are presented for sections of placental tissues treated with $1 \mathrm{mg} /$ $\mathrm{mL}$ PEG-Cy5.5 ( $\sim 200 \mu \mathrm{g} / \mathrm{mL}$ Cy5.5 equiv). The complete data set including all concentrations tested (PEG-Cy5.5 conjugate at $0-1 \mathrm{mg} / \mathrm{mL}, 0-$ $100 \mu \mathrm{g} / \mathrm{mL}$, Cy5.5 equiv) are reported in Figure S2. Individual explants were OCT embedded, frozen, sectioned, and then counterstained with DAPI, a nuclear marker (blue). Placental uptake was assessed by fluorescence microscopy. White arrows are pointing to the syncytiotrophoblast. All images were captured at $20 \times$ magnification. Representative images of $n=3$ experiments.

pathological conditions, ${ }^{25,26}$ which indicates that, as required, PEG did not significantly influence the normal proliferation and apoptosis rates within these tissues.

The potential toxicity of PEG toward the explants was also evaluated by measuring the release of $\mathrm{LDH}$. The amount of $\mathrm{LDH}$ released in the media by the explants is considered as an indicator of necrosis, as $\mathrm{LDH}$ is a biomarker released from the cytoplasm when the cell membrane undergoes lysis. ${ }^{27}$ The results showed no concentration-dependent effects of free PEG on the explants after 24 or $48 \mathrm{~h}$ of incubation. $\mathrm{LDH}$ release did not significantly increase compared to levels observed in untreated control explants $(P>0.05)$ (Figure 4A,B). These results indicated that normal tissue integrity is preserved within the explants and, as required, treatment with PEG did not induce necrosis. $^{28,29}$

Finally, the effect of PEG on the endocrine function of the explants was assessed by quantifying the hCG release into the culture media. hCG is a hormone secreted by the placenta to 
maintain pregnancy. Abnormal levels are usually related to pathological conditions in the mother or the fetus. ${ }^{30}$ Our results revealed that incubation with free PEG had no significant effect on hCG release from the explants at any tested concentration at neither 24 nor $48 \mathrm{~h}$ post-treatment $(P>0.05)$ (Figure 4C,D). These data are in line with other studies evaluating the level of hCG secreted from healthy human placental cells. Impaired implantation and placental development and exposure to exogenous substances, including endocrine disrupting chemicals, have been shown to alter hCG secretion, which at high and low concentrations have been considered as markers of preeclampsia (in late gestation) and miscarriage, respectively. ${ }^{31}$ The normal levels of hCG secretion observed in PEG treated explants suggest no adverse effects of PEG on these tissues.

These findings show that PEG (as a polymeric carrier) did not induce placental dysfunction at histological and physiological levels, which indicate it is suitable for drug delivery systems for use in pregnancy.

PEG Prevents Uptake of the Cy5.5 Dye by the Term Placental Villous Tissues. The confirmed biocompatibility of the free PEG with placental tissues allowed us to proceed with $e x$ vivo (within term placental villous tissue) uptake studies to prove the principle that the uptake of a freely diffusible molecule (Cy5.5) could be prevented via conjugation to PEG using a nonbiodegradable linker.

Explants were incubated with (a) the tumor-homing peptide iRGD-Rho ( $20 \mu \mathrm{M}$; positive control), which is known to freely accumulate within the outer syncytiotrophoblast layer of the placenta, ${ }^{11}$ (b) the free Cy5.5 dye at $1-2 \mu \mathrm{g} / \mathrm{mL}$, or (c) different concentrations ( 0 to $\sim 100 \mu \mathrm{g} / \mathrm{mL}, \mathrm{Cy} 5.5$ equiv) of PEG-Cy5.5 conjugate for 6 or $24 \mathrm{~h}$.

As expected, the iRGD peptide was detected within the outer syncytiotrophoblast layer of the placental villi after $6 \mathrm{~h}$ and was still present after $24 \mathrm{~h}$ of incubation ${ }^{11}$ (Figure 5A). The free Cy5.5 dye was also able to accumulate within the explants at 6 and $24 \mathrm{~h}$ of incubation (Figures $5 \mathrm{~B}$ and S1). In contrast to the free $\mathrm{Cy} 5.5$, the $\mathrm{PEG}-\mathrm{Cy} 5$ conjugate did not show any accumulation in any region of the placental explants after 6 or $24 \mathrm{~h}$ of incubation at any concentration (Figures 5C, S2, and S3). These results confirmed that conjugating Cy5.5 to PEG was successful at preventing the accumulation of Cy5.5 within the tissues. As hypothesized, this effect is likely to be due to a sizeexclusion mechanism, given that others have reported that permeation across the placental barrier is size dependent. ${ }^{32}$

PEG Prevents Uptake of Haloperidol by the Placental Villous Tissues. Having proven that free PEG is biocompatible with human term placental tissue and that conjugation of Cy5.5 to PEG did indeed prevent the fluorophore from being taken up by the explants, we further assessed the strategy by testing a clinically relevant drug. Given that haloperidol can cross the placenta and potentially cause teratogenic effects, ${ }^{33}$ PEGhaloperidol conjugates seemed ideal to be tested within this study.

Placental tissue extracts were incubated for $24 \mathrm{~h}$ with free or conjugated haloperidol at $20 \mu \mathrm{M}$ (haloperidol or haloperidol equiv., a concentration that allowed detection with our RPHPLC method). Unconjugated haloperidol freely diffused into the explants and accumulated at $1.8 \pm 0.51 \mu \mathrm{g} / \mathrm{mg}$ protein (Figure 6). The uptake of the free haloperidol by the placental tissues was expected due to the physicochemical properties (i.e., small size MW and high lipid solubility $)^{10}$ and the probable mechanism involving passive diffusion. ${ }^{34}$ As hypothesized, significantly lower placental levels of the PEG-haloperidol

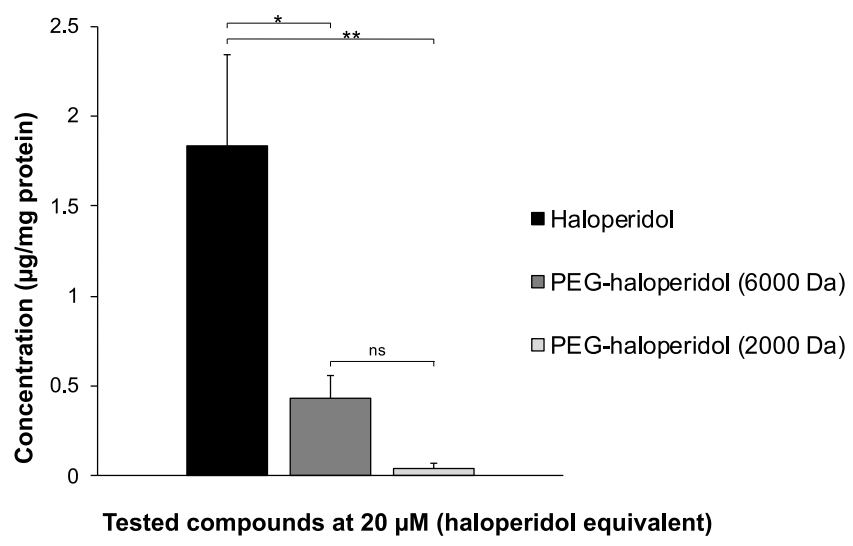

Figure 6. Uptake of free and conjugated haloperidol by placental explants after $24 \mathrm{~h}$ of incubation. Data were normalized to protein contents within the explants. Data were represented as mean \pm SEM $(n$ $=4)$. Data were normally distributed (Shapiro-Wilk test, $P>0.05)(*$ : $P<0.05$, **: $P<0.01$; ns: not significant; one-way ANOVA followed by Bonferroni's post hoc test).

conjugates $(6000$ and $2000 \mathrm{Da})$ were measured at $0.43 \pm 0.13$ and $0.04 \pm 0.03 \mu \mathrm{g} / \mathrm{mg}$ protein $(P<0.05,0.01)$, respectively (Figure 6). The conjugation of haloperidol to the hydrophilic PEG therefore led to the reduced penetration of haloperidol into the placental tissues, as its uptake was reduced by up to $98 \%$ compared to explants exposed to free haloperidol. In the case of PEG-haloperidol (6000 Da), the placental uptake was reduced by $\sim 4$-fold; it was reduced by more than $\sim 40$-fold when PEG (2000 Da) was used within the conjugate. However, there was no significant difference in the uptake levels between the two PEGs $(P>0.05)$. Further work using other in vivo experimental models is required to investigate whether the PEG-haloperidol conjugates taken up by the placenta reach the fetal circulation.

These results are in good agreement with a previous ex vivo study that reported the use of PAMAM-Alexa (488) (a dendrimer-based conjugate) as a model drug delivery system to reduce the transplacental transport of conjugated drugs. ${ }^{4}$ The results revealed that the PAMAM-Alexa $(488)$ conjugate $\left(M_{\mathrm{W}}\right.$ $\sim 16700 \mathrm{Da})$ exhibited a low trans-placental transfer rate when tested using the dually perfused, recirculating term human placental lobule ex vivo model. Despite the absence of PAMAM-Alexa (488) from the capillaries of the villi, the immunohistochemical studies revealed the presence of PAMAM-Alexa at sparse levels in the outer rim of villous branches and in the intervillous spaces. Moreover, the PAMAM conjugate was occasionally detected in the cytoplasm and nuclei of the syncytiotrophoblast, which was not the case for our PEGCy5.5 conjugate, which was not present within the tissues.

It is important to highlight that, for effective treatment of the expectant mother with drug delivery systems prepared from PEG-drug conjugates, it is essential to retain the therapeutic activity of the conjugated drug when a non-prodrug conjugate is developed. This is because the activity of the system does not depend on the release of the conjugated drug. We have previously proven that PEG-conjugated haloperidol maintained its therapeutic effects via different receptors in vitro, however, at a lower extent when compared to the free haloperidol. ${ }^{10}$ These results indicate the suitability of our PEG-drug conjugates as non-prodrug systems to design and develop drug delivery vehicles that could provide compartmentalized therapeutic effects to pregnant women without affecting the developing fetus. 


\section{CONCLUSION}

In this study, an ex vivo tissue culture was employed to demonstrate the utility of PEG-drug conjugates as non-prodrug systems to reduce the uptake of drugs by the placenta. The approach evaluated (a) the biocompatibility of PEG (as a carrier) with the placental tissues and (b) the ability of PEGylated systems to reduce the uptake of conjugated drugs into placental tissue. The study revealed that, as required, PEG did not significantly influence the basal rate of proliferation, apoptosis, or necrosis in human placental explants. Moreover, no modulation of the hCG secretion from the placental explants was recorded after exposure to free PEG, which suggests that PEG does not negatively affect the normal tissue function. With regard to the required reduced uptake into the placenta, in contrast to unconjugated Cy5.5, no PEG-conjugated Cy5.5 was detected within the placental explants at any of the concentrations examined. This finding was further evidenced by measuring the placental accumulation of PEG-haloperidol conjugates, where the conjugation of haloperidol to PEG reduced the tissue concentration of haloperidol up to 40-fold. Together, these results indicate that the non-prodrug design of the PEG conjugates is a feasible approach to reduce or even prevent the transfer of drugs across the placenta. Therefore, the careful selection of drugs and proper design of the linker could allow localization of the therapeutic effects of the conjugated drug within the maternal circulation, while protecting the fetus from any harmful effects of the drug.

\section{ASSOCIATED CONTENT}

\section{(5) Supporting Information}

The Supporting Information is available free of charge at https://pubs.acs.org/doi/10.1021/acs.molpharmaceut.1c00498.

Images of the accumulation of free Cy5.5 dye in the placental explants and the absence of the PEG-Cy5.5 conjugate from the placental explants (PDF)

\section{AUTHOR INFORMATION}

\section{Corresponding Authors}

Francesca Greco - Reading School of Pharmacy, University of Reading, Reading RG6 6AD, United Kingdom; @ orcid.org/ 0000-0001-7934-0056; Email: f.greco@reading.ac.uk

Lynda K. Harris - Maternal and Fetal Health Research Centre, School of Medical Sciences, University of Manchester, St. Mary's Hospital, Manchester M13 9WL, United Kingdom; St. Mary's Hospital, Manchester Foundation Trust, Manchester Academic Health Science Centre, Manchester M13 9WL, United Kingdom; Division of Pharmacy and Optometry, School of Health Sciences, The University of Manchester, Manchester M13 9PL, United Kingdom;

Email: lynda.k.harris@manchester.ac.uk

\section{Authors}

Abbie Dodd - Maternal and Fetal Health Research Centre, School of Medical Sciences, University of Manchester, St. Mary's Hospital, Manchester M13 9WL, United Kingdom; St. Mary's Hospital, Manchester Foundation Trust, Manchester Academic Health Science Centre, Manchester M13 9WL, United Kingdom; Present Address: Nanomedicine Lab, School of Medical Sciences, The University of Manchester, Oxford Road, Manchester M13 9PL, United Kingdom
Az Alddien Natfji - Reading School of Pharmacy, University of Reading, Reading RG6 6AD, United Kingdom

Angelos Evangelinos - Maternal and Fetal Health Research Centre, School of Medical Sciences, University of Manchester, St. Mary's Hospital, Manchester M13 9WL, United Kingdom; St. Mary's Hospital, Manchester Foundation Trust, Manchester Academic Health Science Centre, Manchester M13 9WL, United Kingdom

Antonella Grigoletto - Department of Pharmaceutical and Pharmacological Sciences, University of Padova, 35100 Padova, Italy

Gianfranco Pasut - Department of Pharmaceutical and Pharmacological Sciences, University of Padova, 35100 Padova, Italy; (i) orcid.org/0000-0002-8754-0899

Frances Beards - Maternal and Fetal Health Research Centre, School of Medical Sciences, University of Manchester, St. Mary's Hospital, Manchester M13 9WL, United Kingdom; St. Mary's Hospital, Manchester Foundation Trust, Manchester Academic Health Science Centre, Manchester M13 9WL, United Kingdom; Division of Pharmacy and Optometry, School of Health Sciences, The University of Manchester, Manchester M13 9PL, United Kingdom

Lewis Renshall - Maternal and Fetal Health Research Centre, School of Medical Sciences, University of Manchester, St. Mary's Hospital, Manchester M13 9WL, United Kingdom; St. Mary's Hospital, Manchester Foundation Trust, Manchester Academic Health Science Centre, Manchester M13 9WL, United Kingdom; Division of Pharmacy and Optometry, School of Health Sciences, The University of Manchester, Manchester M13 9PL, United Kingdom

Helen M. I. Osborn - Reading School of Pharmacy, University of Reading, Reading RG6 6AD, United Kingdom

Complete contact information is available at:

https://pubs.acs.org/10.1021/acs.molpharmaceut.1c00498

\section{Author Contributions \\ ${ }^{\ddagger}$ A.D., A.A.N., and A.E. have equally contributed to this work. Notes}

The authors declare no competing financial interest.

Data availability: All data are available from the corresponding authors on reasonable request.

\section{ACKNOWLEDGMENTS}

The authors would like to thank the University of Reading and the Council for At-Risk Academics (Cara) for the academic placement and financial support for A.A.N. as well as the University of Reading Research Endowment Trust Fund (RETF) for their funding of the project. The authors would also like to thank the University of Reading for the use of the Chemical Analysis Facility. F.B. and L.R. were funded by The Medical Research Council (MR/P023401/1). The Maternal and Fetal Health Research Centre is supported by funding from Tommy's the Baby Charity, an Action Research Endowment Fund, the Manchester Biomedical Research Centre, and the Manchester Academic Health Science Centre.

\section{ABBREVIATIONS}

Cy5.5, cyanine-5.5; hCG, human chorionic gonadotrophin; iRGD-Rho, placental homing peptide iRGD-rhodamine; LDH, lactate dehydrogenase; PEG, poly(ethylene glycol); RP-HPLC, reversed-phase high performance liquid chromatography; SEC, size exclusion chromatography. 


\section{REFERENCES}

(1) Tetro, N.; Moushaev, S.; Rubinchik-Stern, M.; Eyal, S. The Placental Barrier: The Gate and the Fate in Drug Distribution. Pharm. Res. 2018, 35 (4), 71.

(2) Dathe, K.; Schaefer, C. The Use of Medication in Pregnancy. Dtsch. Arztebl. Int. 2019, 116 (46), 783-790.

(3) Fisk, N. M.; Atun, R. Market Failure and the Poverty of New Drugs in Maternal Health. PLOS Med. 2008, 5 (1), No. e22.

(4) Menjoge, A. R.; Rinderknecht, A. L.; Navath, R. S.; Faridnia, M.; Kim, C. J.; Romero, R.; Miller, R. K.; Kannan, R. M. Transfer of PAMAM Dendrimers across Human Placenta: Prospects of Its Use as Drug Carrier during Pregnancy. J. Controlled Release 2011, 150 (3), 326-338.

(5) Araújo, R. V. de; Santos, S. da S.; Igne Ferreira, E.; Giarolla, J. New Advances in General Biomedical Applications of PAMAM Dendrimers. Molecules 2018, 23 (11), 2849.

(6) Balakrishnan, B.; Nance, E.; Johnston, M. V.; Kannan, R.; Kannan, S. Nanomedicine in Cerebral Palsy. Int. J. Nanomed. 2013, 8, 41834195.

(7) Labieniec-Watala, M.; Watala, C. PAMAM Dendrimers: Destined for Success or Doomed to Fail? Plain and Modified PAMAM Dendrimers in the Context of Biomedical Applications. J. Pharm. Sci. 2015, 104 (1), 2-14.

(8) Florendo, M.; Figacz, A.; Srinageshwar, B.; Sharma, A.; Swanson, D.; Dunbar, G. L.; Rossignol, J. Use of Polyamidoamine Dendrimers in Brain Diseases. Molecules 2018, 23 (9), 2238.

(9) Heath, F.; Newman, A.; Clementi, C.; Pasut, G.; Lin, H.; Stephens, G. J.; Whalley, B. J.; Osborn, H. M. I.; Greco, F. A Novel PEGHaloperidol Conjugate with a Non-Degradable Linker Shows the Feasibility of Using Polymer-Drug Conjugates in a Non-Prodrug Fashion. Polym. Chem. 2016, 7 (47), 7204-7210.

(10) Natfji, A. A.; Nikitin, D. O.; Semina, I. I.; Moustafine, R. I.; Khutoryanskiy, V. V.; Lin, H.; Stephens, G. J.; Watson, K. A.; Osborn, H. M. I.; Greco, F. Conjugation of Haloperidol to PEG Allows Peripheral Localisation of Haloperidol and Eliminates CNS Extrapyramidal Effects. J. Controlled Release 2020, 322, 227-235.

(11) King, A.; Ndifon, C.; Lui, S.; Widdows, K.; Kotamraju, V. R.; Agemy, L.; Teesalu, T.; Glazier, J. D.; Cellesi, F.; Tirelli, N.; et al. Tumor-Homing Peptides as Tools for Targeted Delivery of Payloads to the Placenta. Sci. Adv. 2016, 2 (5), No. e1600349.

(12) Harris, L. K.; Crocker, I. P.; Baker, P. N.; Aplin, J. D.; Westwood, M. IGF2 Actions on Trophoblast in Human Placenta Are Regulated by the Insulin-like Growth Factor 2 Receptor, Which Can Function as Both a Signaling and Clearance Receptor. Biol. Reprod. 2011, 84 (3), 440-446.

(13) Matos, P.; Horn, J. A.; Beards, F.; Lui, S.; Desforges, M.; Harris, L. K. A Role for the Mitochondrial-Associated Protein P32 in Regulation of Trophoblast Proliferation. Mol. Hum. Reprod. 2014, 20 (8), 745-755.

(14) Beards, F.; Jones, L. E.; Charnock, J.; Forbes, K.; Harris, L. K. Placental Homing Peptide-MicroRNA Inhibitor Conjugates for Targeted Enhancement of Intrinsic Placental Growth Signaling. Theranostics 2017, 7 (11), 2940-2955.

(15) Cureton, N.; Korotkova, I.; Baker, B.; Greenwood, S.; Wareing, M.; Kotamraju, V. R.; Teesalu, T.; Cellesi, F.; Tirelli, N.; Ruoslahti, E.; et al. Selective Targeting of a Novel Vasodilator to the Uterine Vasculature to Treat Impaired Uteroplacental Perfusion in Pregnancy. Theranostics 2017, 7 (15), 3715-3731.

(16) Igarashi, K.; Kasuya, F.; Fukui, M.; Usuki, E.; Castagnoli, N. Studies on the Metabolism of Haloperidol (HP): The Role of CYP3A in the Production of the Neurotoxic Pyridinium Metabolite HPP+ Found in Rat Brain Following Ip Administration of HP. Life Sci. 1995, 57 (26), 2439-2446.

(17) Gong, Y.-J.; Zhang, X.-B.; Mao, G.-J.; Su, L.; Meng, H.-M.; Tan, W.; Feng, S.; Zhang, G. A Unique Approach toward Near-Infrared Fluorescent Probes for Bioimaging with Remarkably Enhanced Contrast. Chem. Sci. 2016, 7 (3), 2275-2285.
(18) Cooper, M. E.; Gregory, S.; Adie, E.; Kalinka, S. PH-Sensitive Cyanine Dyes for Biological Applications. J. Fluoresc. 2002, 12 (3), 425-429.

(19) Alfaifi, A. A.; Heyder, R. S.; Bielski, E. R.; Almuqbil, R. M.; Kavdia, M.; Gerk, P. M.; da Rocha, S. R. P. Megalin-Targeting Liposomes for Placental Drug Delivery. J. Controlled Release 2020, 324, 366-378.

(20) Aagaard, K. M.; Lahon, A.; Suter, M. A.; Arya, R. P.; Seferovic, M. D.; Vogt, M. B.; Hu, M.; Stossi, F.; Mancini, M. A.; Harris, R. A.; et al. Primary Human Placental Trophoblasts Are Permissive for Zika Virus (ZIKV) Replication. Sci. Rep. 2017, 7 (1), 41389.

(21) Ma, W.; Li, G.; Wang, J.; Yang, W.; Zhang, Y.; Conti, P. S.; Chen, K. Vivo NIRF Imaging-Guided Delivery of a Novel NGR-VEGI Fusion Protein for Targeting Tumor Vasculature. Amino Acids 2014, 46 (12), $2721-2732$.

(22) Harris, J. M.; Chess, R. B. Effect of Pegylation on Pharmaceuticals. Nat. Rev. Drug Discovery 2003, 2, 214.

(23) Sastry, B. V. Placental Toxicology: Tobacco Smoke, Abused Drugs, Multiple Chemical Interactions, and Placental Function. Reprod., Fertil. Dev. 1991, 3 (4), 355-372.

(24) Figueroa-Espada, C. G.; Hofbauer, S.; Mitchell, M. J.; Riley, R. S. Exploiting the Placenta for Nanoparticle-Mediated Drug Delivery during Pregnancy. Adv. Drug Delivery Rev. 2020, 160, 244-261.

(25) Kaya, B.; Nayki, U.; Nayki, C.; Ulug, P.; Oner, G.; Gultekin, E.; Yildirim, Y. Proliferation of Trophoblasts and Ki67 Expression in Preeclampsia. Arch. Gynecol. Obstet. 2015, 291 (5), 1041-1046.

(26) Baker, B. C.; Heazell, A. E. P.; Sibley, C.; Wright, R.; Bischof, H.; Beards, F.; Guevara, T.; Girard, S.; Jones, R. L. Hypoxia and Oxidative Stress Induce Sterile Placental Inflammation in Vitro. Sci. Rep. 2021, 11 (1), 7281 .

(27) Chan, F. K.-M.; Moriwaki, K.; De Rosa, M. J. Detection of Necrosis by Release of Lactate Dehydrogenase Activity. Methods Mol. Biol. 2013, 979, 65-70.

(28) Collett, G. P.; Redman, C. W.; Sargent, I. L.; Vatish, M. Endoplasmic Reticulum Stress Stimulates the Release of Extracellular Vesicles Carrying Danger-Associated Molecular Pattern (DAMP) Molecules. Oncotarget 2018, 9 (6), 6707-6717.

(29) Li, M.; Wu, X.; An, P.; Dang, H.; Liu, Y.; Liu, R. Effects of Resveratrol on Autophagy and the Expression of Inflammasomes in a Placental Trophoblast Oxidative Stress Model. Life Sci. 2020, 256, 117890 .

(30) Costa, M. A. The Endocrine Function of Human Placenta: An Overview. Reprod. BioMed. Online 2016, 32 (1), 14-43.

(31) Paulesu, L.; Rao, C. V.; Ietta, F.; Pietropolli, A.; Ticconi, C. HCG and Its Disruption by Environmental Contaminants during Human Pregnancy. Int. J. Mol. Sci. 2018, 19, 914.

(32) Aengenheister, L.; Keevend, K.; Muoth, C.; Schönenberger, R.; Diener, L.; Wick, P.; Buerki-Thurnherr, T. An Advanced Human in Vitro Co-Culture Model for Translocation Studies across the Placental Barrier. Sci. Rep. 2018, 8 (1), 5388.

(33) Kopelman, A. E.; McCullar, F. W.; Heggeness, L. Limb Malformations Following Maternal Use of Haloperidol. JAMA 1975, 231 (1), 62-64.

(34) Audus, K. L. Controlling Drug Delivery across the Placenta. Eur. J. Pharm. Sci. 1999, 8 (3), 161-165. 